\title{
Estado actual del tratamiento del carcinoma del ovario
}

\author{
POR EL DOCTOR RICARdo RUEda G.
}

Ex-Interno del Servicio de Clínica Ginecológica de la Universidad Nacional.

El cáncer del ovario se presenta con una frecuencia mayor de la que habitualmente se cree, pero su existencia ha sido subestimada como resultado de la incidencia mayor de otros cánceres del aparato genital femenino tales como los carcinomas del cuerpo y del cuello uterinos. Además, el tratamiento activo del carcinoma del ovario se ve en la mayoría de los casos demorado, ya que este órgano está profundamente situado en la pélvis, sin contacto inmediato con alguno otro, cuya función pueda transtornarse fácilmente, llegando en esta forma a estados muy avanzados sin que su presencia se manifieste.

Cuando se descubre precozmente alguna lesión maligna del ovario es, en la mayor parte de los casos, el resultado de una exploración ginecológica rutinaria, el hallazgo en una laparotomía realizada con otro propósito o el resultado de una peculiaridad patológica especial, tal como el desarrollo de una pequeña zona maligna dentro de un quiste por otra parte benigno.

Es interesante anotar que los estudios recientes de Munel (1), indican que no existe relación alguna entre la duración de los sintomas consignados en la historia de la paciente con carcinoria del ovario y la extensión de la enfermedad encontrada al momento de la intervención, anotación que vale la pena tener presente siempre que se piense realizar una intervención encamirada a la extirpación de un blastoma ovárico maligno.

Ahora bien: cada una de las variedades de cáncer ovárico tiene un curso clínico potencial diferente, requiriendo por lo tanto una consideración especial en cuanto se refiere a su tratamiento.

Los cistadenomas serosos papilares, los pseudomucinosos, etc., constituyen cada uno una enfermedad y presentan también probiemas terpéuticos diferentes. 
Otro problema terapéutico depende de una consideración anatomopatológica especial, es decir, si existen o no lesiones precancerosas del ovario. Este término de lesión precancerosa o subclinica se ha hecho común entre los ginecólogos cuando se trata de explotar el tema de la leucoplasia vulvar, del carcinoma intraepitelial del cuello o de la hiperplasia endometrial. Parece casi seguro como dice Meigs (2), que en el ovario se pueden producir lesiones similares, pero claro está, muy difíciles de conocer dada la topografía peculiar del gonado femenino, y por ello algunos investigadores entre los que se cuenta especialmente Decker (3), recomiendan el empleo sistemático de la culdoscopia cuando al examen ginecológico se aprecia algún endurecimiento o hipertrofia del ovario sospechosos de lesión maligna. Mediante el empleo del culdoscopio se pueden apreciar los cambios macroscópicos del ovario que, al fin y al cabo, no suministran datos de mucho valor, pero mediante alguno de sus accesorios se pueden tomar biopsias de las lesiones sospechosas para su estudio histológico. Desafortunadamente este método se encuentra aún poco estudiado y es empleado en muy pocos centros, en donde parece por las experiencias publicadas, ha dado resultados muy halagadores en lo que pudiéramos llamar diagnóstico precoz del cáncer del ovario.

Constituye una experiencia frecuente encontrar una lesión maligna localizada en un tumor benigno como lo anotamos anteriormente, hallazgos que sugieren que existe una tendencia en muchos tumores ováricos a que se desarrolle un tumor maligno en su tejido, hecho que confirma la opinión de muchos patólogos, quienes sostienen que todas las neoformaciones ováricas están en relación muy cercana con la malignidad y que en ocasiones puecen ser origen de un cáncer, sea por la aparición de zonas malignas dentro de los mismos o por un cambio de estructura hacia la forma maligna, opiniones que deben recordarse al realizar el tratamiento de los tumores ováricos aparentemente benignos.

Problema de la profilaxis del cáncer del ovario.- El chequeo gınecológico de toda mujer que pase de los 35 años a intervalos de seis meses a un año tiene como uno de sus objetivos el reconocimiento precoz del cáncer del ovario, y debe constituir el primer paso para poder hablar posteriormente de curación. Pero la práctica de este sistema no la podemos aún calificar de buena por varios factores: en primer lugar, y especialmente en nuestro medio, las mujeres no acuden a la consulta del especialista 
sino cuando se dan cuenta que son portadoras de una gran tumoración pélvica o cuando un derrame ascítico ha alcanzado tales proporciones que les impide llevar una vida normal. En seEundo lugar, las dificultades de descubrir un pequeño tumor por los métodos aproximados del examen bimanual son en ocasiones considerables. Sin embargo son de recomendar tales exámenes rutinarios, ya que las estadísticas de speert calculan que casi un na mujer de cada cien que llegan a los cuarenta años muere de cáncer del ovario. Ahora bien: el tratamiento adecuado del engrosamiento del ovario hallado en el examen bimanual presenta también un problema en la prevención del cáncer del ovario, ya çue no son infrecuentes los engrosamientos menores del ovario por congestión, edema o quistes foliculares de retención que no justifican una laparotomía. Por ello en estos casos puede uno guiarse por un plan general establecido por Taylor, citado por Meigs, "todo engrosamiento de más de $5 \mathrm{~cm}$. de diámetro en una mujer por debajo de los cuarenta años deberá ser observado a intervalos de dos a tres meses, hasta que se observe que el tamaño del ovario disminuya, o por lo menos no aumente. Por otra parte, los engrosamientos progresivos del ovario más allá del límite general de $5 \mathrm{~cm}$. en mujeres jóvenes, y cualquier engrosamiento significativo en mujeres después de la menopausia, debe constituir una indicación para la exploración abdominal".

La posibilidad de prevenir el desarrollo ulterior de un carcinoma ovárico mediante la exploración rutinaria de los ovarios en operaciones pélvicas realizadas por otros motivos, también existe. Speert, en un estudio de 260 mujeres con tumores malignos del ovario, encontró que el $26 \%$ habían sido sometidas a intervenciones ginecológicas previas, cifras que se aproximan a las de Montgomery (4), quien encontró un $21 \%$.

Ya habíamos anotado que cada uno de los blastomas ováricos representa una entidad patológica diferente y que por consiguiente su tratamiento será distinto en cada caso especial.

Dado que una parte importante del grupo considerado como carcinoma ovárico no es primitivo sino metastásico de algún otro origen, hay necesidad de hacer estudios complementarios preoperatorios de posibles localizaciones primitivas en otros lugares. La historia cuidadosa y el examen físico excluirán o no la presencia del tumor primitivo en el séno, útero o tubo gastro-intestinal, y como regla general debe practicarse el estudio radiológico gas- 
tro-intestinal en busca de un posible tumor primario de estómago o cólon.

Estos tumores son considerados como incurables ya que, cuando aparecen, el tumor primitivo ha alcanzado grandes proporciones y ha hecho metástasis a otros órganos. Sin embargo se acostumbra hacer su extirpación cuando el estado general de la enferma lo permite con la única finalidad de aliviar las molestias abdominales. Es el caso del tumor de Krukenberg.

En el tratamiento de los tumores ováricos malignos existen en la actualidad dos procedimientos: el quirúrgico y el fisioterápico y tres son los períodos de su tratamiento en que es preciso llegar a una decisión respecto al tratamiento que ha de empiearse:

1) Cuando se ve por primera vez a la enferma, determinar si ha de practicarse la operación. 2) Una vez abierta la cavidad abdominal decidir el tipo de operación y la amplitud de la misma, y 3) Después de la operación resolver si se ha de emplear fisioterapia.

En cuanto a lo primero, se puede decir que muchas veces no se hace el diagnóstico de tumor ovárico maligno hasta después de la laparatomía y por lo tanto es preciso admitir que en los casos dudosos se impone la exploración quirúrgica.

La magnitud y el tipo de operación que se debe realizar se cefine como lo anotamos con la cavidad abdominal abierta y variará para cada caso particular, pero se tendrán en cuenta siempre algunas normas generales. En primer lugar, las vías de propagación y las primeras estaciones, más comunes, de las metástasis. La extensión linfática tiene lugar hasta cierto grado y en los casos avanzados se pueden encontrar metástasis en los ganglios retroperitoneales a lo largo de los grandes vasos. Sin embargo, deben considerarse otras dos vías de propagación: la transplantación de células a través de la trompa que explica la frecuencia de las lesiones encontradas en el endometrio, y el transporte activo de células viables localizadas en el ovario a través de la cavidad peritoneal hasta diversas partes del peritoneo, implantes que se encuentran más frecuentemente sobre las asas intestinales en contacto con la pélvis y más a menudo en el epiplón rnayor.

Luego de realizada la laparotomía se observará la presencia - ausencia del líquido ascítico y sus características, ya que el rallazgo de sangre en éste habla más en favor de la extensión 
cle la enfermedad que la misma cantidad, indicando en el primer caso que la neoplasia ha traspasado los límites de la cápsula; en cambio el segundo puede presentarse en tumores benignos como en el caso del fibroma ovárico (Sindrome de Meigs).

Luego de inspeccionar las tumoraciones ováricas, se decidirá sobre la conducta que se debe seguir en cuanto a su extirpación, y así tenemos que en algunas oportunidades se impone la resección de alguna porción de una víscera hueca junto con el blastoma. El problema sobre el tratamiento que debe darse al ovario restante se debe resolver en el mismo acto quirúrgico. Quizás la mayoría de los cánceres del ovario, dice Meigs, son procesos bilaterales y por lo tanto no se presenta alguna vacilación. Pero cuando el ovario opuesto es aparentemente normal y la mujer es joven, se puede vacilar en la conducta.

Los cistadenomas serosos papilares y los cistadenocarcinomas por ejemplo, presentan gran tendencia al desarrollo bilateral y por lo tanto, es necesaria la extirpación del ovario contralateral aunque aparentemente sea normal. La extirpación del útero está también indicada en todos los casos operables de carcinoma ovárico si se recuerda que a través de las trompas se pueden hacer frecuentemente metástasis.

Radioterapia- - Para la administración científica y eficaz de la radioterapia, como en el caso de la cirugía, es necesario tener conocimiento de la distribución anatómica de la lesión primaria y de sus metástasis más comunes.

Dada la extensión del carcinoma ovárico a través del peritoneo, vehiculizado por el líquido ascítico, se tendrá presente la posibilidad de metástasis no solamente en el abdomen inferior sino también en el superior, campo que deberá omitirse en los casos en que el cirujano está seguro de la limitación del proceso al abdomen inferior, ya que la irradiación sobre las áreas hepáticas y esplénica, causa gran malestar a más de no encontrarse exenta de peligros.

La radioterapia se aplica generalmente en el post-operatorio, y el hecho de la mejoría dramática en gran número de casos es el mejor argumento en pro del sistema; usualmente se administra tan pronto como el estado general de la enferma lo permita, empezándose desde una semana hasta tres después de la operación.

Hay sin embargo, ocasiones en que la radioterapia se emplea en el preoperatorio, ya que no son pocos los casos en que el sis- 
tema hace posible la intervención, disminuyendo el tamaño de la lesión y sus metástasis.

Ahora bien, la sensibilidad de los tumores malignos del ovario varía según su tipo histológico. Entre los más sensibles se encuentra el disgerminoma, similar del seminoma del testículo. Los tumores de la granulosa muestran generalmente gran resistencia. La sensibilidad de los cistadenomas papilares es variable, aunque hay la creencia de que existe mayor sensibilidad en aquellos que tienen cierto grado de calcificación.

Finalmente queremos incluir la lista de los tumores malignos del ovario en que se encuentra indicado alguno de los procedimientos anotados, tomada de una de las clasificaciones más aceptadas por nuestra escuela (5).

\section{Epiteliales malignos}

a) Carcinoma primitivo. Quístico: cistadenocarcinoma seroso papilar, seudomucoso, etc.

Tratamiento quirúrgico: resección del ovario afectado y radiación postoperatoria en mujeres jóvenes. Resección del ovario contralateral e histerectomía total en premenopaúsicas y menopáusicas, radiación postoperatoria.

b) Carcinomas metastásicos.

Tratamiento quirúrgico cuando las condiciones generales de la enferma lo permitan con la única finalidad de aliviar las molestias pélvicas.

Conjuntivos malignos

a) Sarcoma primitivo.

Radioterapia preoperatoria, cirugía radical y radiación postoperatoria.

b) Sarcoma secundario, a melanoma cutáneo, etc.

Tratamiento quirúrgico cuando el estado de la enferma lo permita.

\section{Displásicos malignos}

\section{Feminizantes}

a) Foliculomas. Teca y granulosa.

Tratamiento quirúrgico conservador, ya que rara vez dan metástasis, radical cuando lo hacen. Recordar, la concomitancia de los tumores de granulosa con el carcinoma endometrial. 


\section{Masculinizantes}

a) Masculinovoblastoma, tumor ovárico de aspecto adrenal cue rara vez da metástasis. Cuando no hay, cirugía conservadora. Cuando hay, cirugía radical.

b) Arrenoblastoma. Es en ocasiones maligno (variedad sarcomatosa). Cuando ha sobrepasado la cápsula está justificado el sacrificio del útero, trompas y ovario contralateral.

Néutros

a) Disgerminoma. Similar del seminoma del testículo. En este tipo de tumor se impone la cirugía radical, ya que la extirpación local da un $80 \%$ de recidivas. Tanto la lesión primitiva como las metástasis son altamente sensibles a los rayos $\mathrm{x}, \mathrm{y}$ por ello beneficia la radioaterapia postoperatoria.

\section{BIBLIOGRAFIA}

1. MUNEL, citados por Crossen y Crossen, "Enfermedades de la mujer". IX. edición. Fág. 781. Uthea. Méjico, 1949.

2. MEIGS J. V. y STURGIS S. H.- "Progresos de la Ginecología" II. edición. Pág. 545. Científico Médica. Barcelona. 1953.

3. DECKER, ALBERT.- Culdoscopy. 1a edition. Pág. 230. Saunders. London, 1951.

4. MONIGOMERY, JHON.- Ovarian Crcinoma. Eulletin of the new England Medical Center. 1: 6. February, 1954.

5. LOPEZ E. GUILLERMO.- Conferencias de Ginecología dictadas en el Hospital San Juan de Dios de Bogotá. 1952. 